\title{
The Measurement of Reliability based on the Heterogeneity of the Network
}

\author{
Kai $\mathrm{Li}^{\mathrm{a}}$ and Yongfeng $\mathrm{He}^{\mathrm{b}}$ \\ Academy of Armored Force Engineering Beijing 100072, China \\ accvip0@126.com, bheyfeng@sina.com
}

\begin{abstract}
Keywords: Reliability, Random Network, Scale-Free Network, Heterogeneity.
\end{abstract}
\begin{abstract}
The reliability of complex network is an important content in the research of complex networks. In order to evaluate the reliability of the complex network, the standard deviation of the degree values to characterize the heterogeneity of the network, considering the difference of the degree distribution, such as Poisson distribution and Power-laws distribution in random network and scale-free network, a function on the reliability of the complex network is defined with convergence and monotonicity which is proved. Under two failure models, random destruction and intentional attack, we simulate the reliability function by the proportion of removed edges. Then we analysis the relation between the network heterogeneity and network reliability. The results show that the reliability function is effective and applicable to assess the reliability of complex network.
\end{abstract}

\section{Introduction}

In recent years, complex networks can be seen everywhere in our life, including for the examples such as information, traffic, biology and social activities [1, 2]. At present, the main models are random network model, small-world network model and scale-free network model. Complex networks are usually abstracted into appropriate models instead of complex systems, including a large number of network nodes and complex interconnections between nodes, what's more, the topology of complex networks determine the structure of network properties and function properties. In the study about the real network, many statistical properties such as the shortest average path between network nodes, high clustering coefficient and power-laws degree distribution has been proved [3, 4].The high clustering coefficient and scale-free properties in many real networks could be found, such as the World Wide Web, social networks and logistics networks, etc.

The reliability and survivability of complex networks have been paid more and more attentions [5 6]. The study indicate the random network and scale-free network have diverse characteristics under different nodes fault, and the more heterogeneous of network has, such as the degree distribution, the more reliability to random failure exist, however, the scale-free network with more heterogeneous shows higher fragility by removal of important nodes and edges than random network. The standard deviation of the degree value is a measure of network heterogeneity. It is an important way to analyze the characteristics of the complex network from the point of view of the standard deviation.

In this paper, the definition of the network reliability function is proposed based on the standard deviation of the node degree value of the complex network. The convergence and monotonicity of the network reliability function are proved. The reliability changes of the random network and the scale-free network are studied in the two failure modes. The reliability function is analyzed by simulation, and the results are discussed and analyzed. The results show that the heterogeneity of the network has a great influence on the characteristics of the network.

\section{The complex network model}

The complex network model mainly include four typical model period: regular network, random network [7], small-world network[8] and scale-free network[9]. In order to study the topological structure of the complex network model, experts and scholars define lots of quantities parameters, such as degree and degree distribution, average distance, clustering coefficient, connectivity, density, 
network efficiency and network entropy. For example, degree and degree distributions classify networks into homogeneous model or not, according to their topological structure, mainly consider the degree of almost nodes in the network. The entropy of networks is the measure of the disorder or randomness about degree of network. Random networks and scale-free networks are two typical network models, for the random network itself, the existence of connected edges is completely random and the whole network is homogeneous. The scale-free network considers that the network itself is dynamic, the existence of connected edges is not completely randomized, but meets certain evolutionary rules, and now many critical infrastructure networks (such as the Internet, power and rail networks) meet the scale-free characteristics.

\subsection{ER Model.}

The random network model is completely opposite to the regular network, Erdös and Rényi constructed a random network by adding a certain amounts of connected edges randomly between the pair of nodes. The network model can be analyzed by statistics, when the number of nodes $N \rightarrow+\infty$ in the network, the degree distribution of nodes in the ER network model obeys the Poisson distribution in theory [7]:

$$
p(k)=\frac{\lambda^{k} e^{-\lambda}}{k !}
$$

where $\lambda$ is the average degree of the network.

\subsection{BA Model.}

Barabási and Albert pointed out that the real networks have scale-free properties, which considering the non-stochastic dynamic characteristics of "growth" and "preference" of complex networks. There are few nodes with high degree of scalability in scale and a large number of nodes with low values. This non-uniform structure leads to the distribution of the skewness degree series, which is expressed as the degree distribution of power law[9]:

$$
p(k)=\alpha k^{-q}
$$

where $\alpha$ is the coefficient of the distribution and $q$ is the index of the degree distribution.

\section{The reliability function of complex network}

\subsection{Definition.}

Complex network reliability refers to the network under the specified conditions, within a specified time to complete the specified function. Complex networks usually contain a large number of nodes, the complex interconnections between the heterogeneous nodes called edges of the network, the entire network as a sophisticated model with multi-levels, multi-dimensions and multi-attributes. Without considering the multi-edges between pairs of nodes and self-loop, for the undirected network, the complex network is abstracted as graph $G(V, E)$, where $V=\left\{v_{1}, v_{2}, \cdots v_{N}\right\}$ denotes the set of nodes in the network and $N$ is the amount of the nodes, $E=\left\{e_{1}, e_{2}, \cdots e_{M}\right\}$ denotes the set of edges in the network and $M$ is the amount of the edges. Network topology structure with nodes and edges determine the connectivity of the network which effect the exchange of information flow, material flow and energy flow $[10,11]$. By deleting or adding network edges have important influence on network connectivity. Since the degree and degree distribution of the complex network are the direct reflect of the network topology heterogeneity, we define the network reliability function based on the standard deviation of nodes degree [12].

The network reliability function $H(\sigma, N, M)$ is defined as:

$$
H(\sigma, N, M)=\exp \left\{20\left[\frac{\sigma}{N(N-1)}+\frac{M}{N(N-1)}-\frac{N(N-1)}{2}\right]\right\}
$$

Where $\sigma=\left[\frac{1}{N} \sum_{i=1}^{N}\left(d_{i}-\frac{2 M}{N}\right)^{2}\right]^{\frac{1}{2}}$ stand for the standard deviation of nodes degree, $d_{i}$ is the value of the nodei. 


\subsection{Features of the network reliability function}

According to the definition of the reliability of complex network, the network reliability function $H(\sigma, N, M)$ suit two features:

(1) Convergence: the function is between 0 and 1.

(2) Monotonicity: delete one edge of the network, the value of $H(\sigma, N, M)$ will reduce.

We prove the two features as follows:

(1)Obviously, the $H(\sigma, N, M)$ is one exponential function, thus:

$$
\begin{aligned}
0<H(\sigma, N, M) & =\exp \left\{20\left[\frac{\sigma}{N^{2}(N-1)}+\frac{M}{N(N-1)}-\frac{1}{2}\right]\right\} \\
& \leq \exp \left\{20\left[\frac{d_{\max }-d_{\min }}{N^{2}(N-1)}+\frac{M}{N(N-1)}-\frac{1}{2}\right]\right\} \\
& \leq \exp \left\{20\left[\frac{N-1-0}{N^{2}(N-1)}+\frac{M_{\max }}{N(N-1)}-\frac{1}{2}\right]\right\}=1
\end{aligned}
$$

Where $M_{\text {max }}=0.5 N(N-1)$, the network is globally coupled network under the present circumstances. The degree of each node in the network meet $d=N-1$, the standard deviation of nodes degree $\sigma=0$. The reliability of globally coupled network is $H(\sigma, N, M)=1$, since any two nodes of globally coupled network are connected directly, the network has the highest reliability.

In another extreme case, the network contains $N$ nodes, the amount of the edges is $M=0$, there is no edges in the network called isolated point network. To calculate the reliability of the network, the results are: the standard deviation of nodes degree $\sigma=0$, the reliability of isolated point network is:

$$
H(\sigma, N, M)=\exp (-10)=4.54 \times 10^{-5} \approx 0
$$

With the above analysis about the convergence of the function, we prove $H(\sigma, N, M)$ satisfy $0 \leq H(\sigma, N, M) \leq 1$

(2) We assume $G^{\prime}\left(V, E^{\prime}\right)$ is obtained from $G(V, E)$ by deleting one edge, if we want to prove the monotonicity, we should demonstrate $H^{\prime}(\sigma, N, M-1)<H(\sigma, N, M)$.

We assume the two nodes of the deleted edge are $i_{0}$ and $i_{1}$, in the graph $G^{\prime}\left(V, E^{\prime}\right)$, if $i \neq i_{0}$ and $i \neq i_{1}$,

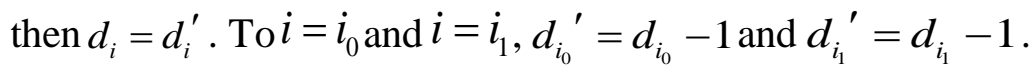

As $0 \leq H(\sigma, N, M) \leq 1$, if we want to prove $H^{\prime}(\sigma, N, M-1)<H(\sigma, N, M)$, we need to certify:

$$
\frac{H^{\prime}\left(\sigma^{\prime}, N, M-1\right)}{H(\sigma, N, M)}<1
$$

If $\frac{H^{\prime}\left(\sigma^{\prime}, N, M-1\right)}{H(\sigma, N, M)}<1$, equal $\sigma^{\prime 2}<(\sigma+N)^{2}$.

The left of the inequality $\sigma^{\prime 2}$ is:

$$
\begin{aligned}
& \sigma^{\prime 2}=\frac{\sum_{i=1}^{N}\left[d_{i}^{\prime}-\frac{2(M-1)}{N}\right]^{2}}{N}=\frac{\sum_{i=1}^{N}\left[d_{i}^{\prime 2}\right]}{N}-\frac{4(M-1)}{N^{2}} \sum_{i=1}^{N}\left[d_{i}^{\prime}\right]+\frac{4(M-1)^{2}}{N^{2}} \\
= & \frac{\sum_{i=1, i, i i_{i}, i_{i}}^{N}\left[d_{i}{ }^{2}\right]}{N}-\frac{4(M-1)}{N^{2}} \sum_{i=1, i, i_{0}, i_{1}}^{N}\left[d_{i}\right]+\frac{\left(d_{i_{0}}-1\right)^{2}}{N}+\frac{\left(d_{i_{1}}-1\right)^{2}}{N}-\frac{4(M-1)\left(d_{i_{0}}+d_{i_{1}}\right)}{N^{2}}+\frac{4(M-1)^{2}}{N^{2}} \\
= & \frac{\sum_{i=1, i \neq i_{0}, i_{i}}^{N}\left[d_{i}{ }^{2}\right]}{N}-\frac{4(M-1)}{N^{2}} \sum_{i=1, i i_{0}, i_{i}}^{N}\left[d_{i}\right]+\frac{4(M-1)^{2}}{N^{2}}+\frac{d_{i_{0}}{ }^{2}+d_{i_{i}}{ }^{2}}{N}-\frac{2\left(d_{i_{0}}+d_{i_{1}}\right)}{N}+\frac{2}{N}-\frac{4(M-1)\left(d_{i_{0}}+d_{i_{1}}\right)}{N^{2}}+\frac{4(M-1)^{2}}{N^{2}}
\end{aligned}
$$

The right of the inequality $(\sigma+n)^{2}$ is: 


$$
\begin{aligned}
& \begin{array}{l}
(\sigma+n)^{2}= \\
\quad \frac{\sum_{i=1}^{N}\left[d_{i}{ }^{2}\right]}{N}-\frac{4 M}{N^{2}} \sum_{i=1}^{N}\left[d_{i}\right]+\frac{4 M^{2}}{N^{2}}+N^{2}+2 n \sigma \\
\quad=\frac{\sum_{i=1, i \neq i_{i}, i_{1}}^{N}\left[d_{i}{ }^{2}\right]}{N}-\frac{4 M}{N^{2}} \sum_{i=1, i \neq i_{0}, i_{1}}^{N}\left[d_{i}\right]+\frac{4 M^{2}}{N^{2}}+\frac{d_{i_{0}}{ }^{2}+d_{i_{1}}{ }^{2}}{N}-\frac{4 M\left(d_{i_{0}}+d_{i_{1}}\right)}{N^{2}}+N^{2}+2 n \sigma \\
\text { If } \sigma^{\prime 2}<(\sigma+N)^{2}, \text { then } \\
4 \sum_{i=1, i \neq i_{0}, i_{i}}^{N}\left[d_{i}\right] \\
N^{2}
\end{array}-\frac{2\left(d_{i_{0}}+d_{i_{1}}\right)}{N}+\frac{2}{N}+\frac{4\left(d_{i_{0}}+d_{i_{1}}\right)}{N^{2}}<N^{2}+2 n \sigma \\
& \frac{4 \sum_{i=1}^{N}\left[d_{i}\right]}{N^{2}}-\frac{2\left(d_{i_{0}}+d_{i_{1}}\right)}{N}+\frac{2}{N}<N^{2}+2 n \sigma \\
& \text { The } d_{\max }=N-1, \text { so: } \\
& \frac{4 \sum_{i=1}^{N}\left[d_{i}\right]}{N^{2}}-\frac{2\left(d_{i_{0}}+d_{i_{1}}\right)}{N}+\frac{2}{N}<\frac{4 N(N-1)}{N^{2}}+\frac{2}{N}<N^{2}+2 n \sigma
\end{aligned}
$$

This inequality is exactitude when $N \geq 3$. In other words, to the arbitrary network with $N \geq 3$, we could obtain $\sigma^{\prime 2}<(\sigma+N)^{2}$ and the second feature is proved.

\section{Numerical simulations and analysis}

\subsection{Fault mode.}

For ER random networks and BA scale-free networks, two failure modes are used to remove the edges in the network: random destruction and intentional attack. Random destruction means that there is no fixed removed target, randomly remove the edge in a certain proportion; intentional attack refers to removing the important connection edges by certain strategy.

In this context, we calculate the importance of edges by edge betweeness, the definition about edge betweeness of edge $e_{m}$ as:

$$
B_{m}=\sum_{i \neq i^{\prime}} \frac{n_{i i^{\prime}}^{m}}{g_{i i^{\prime}}} \quad e_{m} \in E, i, i^{\prime} \in V
$$

Where $g_{i i^{\prime}}$ is the number of the shortest path between the nodes ${ }^{i}$ and $i^{\prime}, n_{i i^{\prime}}^{m}$ is the number of the shortest path contain $e_{m}$ between the nodes ${ }^{i}$ and $i^{\prime}$.

\subsection{Simulation results and discussion}

In order to investigate the reliability of complex network under the influence of the random destruction and intentional attack, computer experiments were performed on both ER model and BA model by removing edges gradually, the simulation results are shown in Fig 1.
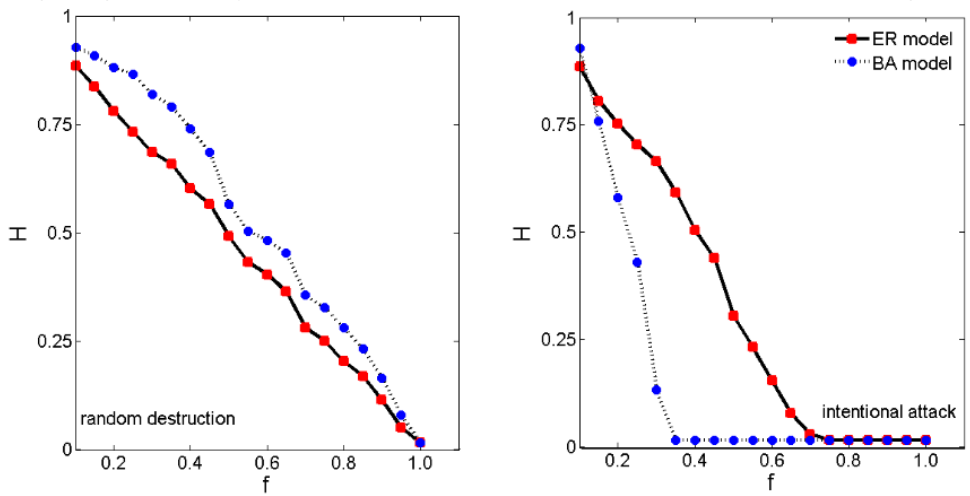

Fig. 1 The reliability function with the proportion of removed edges

In the numerical test we could see the function $H(\sigma, N, M)$ reduce in different ways in ER model and BA model under random destruction and intentional attack according to the removed edges 
proportion $f$. In random failure mode, BA scale-free network shows stronger reliability compared to the ER random network, the curve of the random network descends smoothly than scale-free network, BA network shows greater reliability at $f<0.5$, the results are described that minor perturbations do not affect the reliability of scale-free networks. In intentional attack mode, the curves of random networks and scale-free networks fall very rapidly, scale-free networks are faster than random networks, this indicates that deliberate attacks are more damaging to the network than random failure mode.

In stochastic failure mode, BA scale-free networks show high reliability, mainly because the degree distribution of BA-scale-free networks is power-law distribution with strong non-uniformity: there are a few network Hub nodes. When the proportion is small, the Hub nodes in the network are still connected and the reliability of the network is high. When the proportion of the nodes increases gradually, the Hub nodes begin to descend sharply, which directly reflects the decrease of the network reliability. Because of this non-uniformity, the connection edge of the Hub node becomes the target of deliberate attack. For ER networks, its degree distribution is Poisson distribution, the network reflects the uniformity of the random damage and intentional attack In the two models, the reliability of the ER stochastic network decreases without fluctuation and is relatively even and stable.

\section{Conclusion}

This paper investigates the relationship between the standard deviation and the network reliability in ER random networks and BA scale-free networks under two failure modes, random destruction and intentional attack. By the simulations, we find the topology heterogeneity affect the reliability of the network, completely random and uniform structure is easier to destroy than scale-free structure under random destruction and the scale-free network has strong reliability, however, the Hub nodes as the attacked target under intentional attack and BA model perform vulnerability obviously.

\section{References}

[1]. Albert R, Barabási A. Statistical mechanics of complex networks [J]. Reviews of Modern Physics. Vol. 74 (2002) No. 1, p. 47-97.

[2]. Holme P, Kim B J, Yoon C N, et al. Attack vulnerability of complex networks [J]. Physical Review E. Vol. 65 (2002) No. 5, p. 634-648.

[3]. Min O Y, Pan ZZ, Hong L, et al. Correlation analysis of different vulnerability metrics on power grids[J]. Physica A. Vol. 396 (2014) No. 2, p. 204-211.

[4]. Ghedini C G, Carlos H C. Rethinking failure and attack tolerance assessment in complex networks [J]. Physica A. Vol. 390 (2011) No. 23, p. 4684-4691.

[5]. Zhang J H, Xu X M, Hong L, et al. Attack vulnerability of self-organizing networks [J]. Safety science. Vol. 50 (2012) No. 3, p. 443-447.

[6]. Hu P, Fan W L, Mei S W. Identifying node importance in complex networks [J]. Physica A. Vol. 429 (2015) No. 3, p. 169-176.

[7]. Erdös P, Rényi A. On random graphs [J]. Publicationes mathematicae. Vol. 3 (1956) No. 6, p. 290-297.

[8]. Watts D J, Strogatz S H. Collective dynamics of 'small-world' networks [J]. Nature. Vol. 393 (1998) No. 6684, p. 440-442.

[9]. Barabási A L, Albert R. Emergence of scaling in random networks [J]. Science. Vol. 286 (1999) No. 15 , p. 509-512.

[10].Newman M. The structure and function of complex network [J]. SIAM review. Vol. 45 (2003) No. 2, p. 167-256.

[11].Albert R, Jeong H, Barabási A L. Attack and error tolerance of complex networks [J].Nature. Vol. 406 (2000) No. 6794, p. 378-382.

[12]. Newman M, Strogatz S H, Watts D J. Random graphs with arbitrary degree distributions and their applications [J]. Physical Review E. Vol. 64 (2001) No. 2, p. 212-228. 